\title{
Friction and Wear Characteristics of C/Si Bi-layer Coatings Deposited on Silicon Substrate by DC Magnetron Sputtering
}

\author{
Oleksiy V. Penkov • Yegor A. Bugayev • \\ Igor Zhuravel • Valeriy V. Kondratenko • \\ Auezhan Amanov • Dae-Eun Kim
}

Received: 26 March 2012 / Accepted: 26 June 2012/Published online: 8 July 2012

(C) Springer Science+Business Media, LLC 2012

\begin{abstract}
The tribological behavior of carbon/silicon bi-layer coatings deposited on a silicon substrate by DC magnetron sputtering was assessed and compared to that of amorphous carbon and silicon coatings. The motivation was to develop a wear resistant coating for silicon using thin layers of amorphous carbon and silicon. Wear tests were conducted by sliding a stainless steel ball against the coating specimens under applied normal loads in the range of $20 \sim 50 \mathrm{mN}$. Results showed that the wear rate of the bi-layer coating was strongly dependent on the ratio of thickness between the carbon and silicon layers. The wear rate of the bi-layer coating with $25 \mathrm{~nm}$ thick carbon and $102 \mathrm{~nm}$ thick silicon layers was about 48 and 20 times lower than that of the single-layer amorphous carbon and amorphous silicon coating, respectively. In addition, the steady-state friction coefficient of the bi-layer coating could be decreased to 0.09 by optimizing the thickness of the layer. Finally, a model for the wear reduction mechanism of the carbon/silicon bi-layer coating was proposed.
\end{abstract}

Keywords Friction - Wear - Bi-layer coating - Carbon · Silicon $\cdot$ Sputtering

O. V. Penkov · A. Amanov · D.-E. Kim ( $\square)$

Center for Nano-Wear, Yonsei University, Seoul 120-749, Korea

e-mail: kimde@yonsei.ac.kr

Y. A. Bugayev · I. Zhuravel · V. V. Kondratenko

National Technical University "KhPI”, Kharkov, Ukraine

D.-E. Kim

Department of Mechanical Engineering, Yonsei University, Seoul 120-749, Korea

\section{Introduction}

Single-layer coatings have been effectively utilized for decades to reduce the friction and wear of mechanical components in various devices [1-3]. Considering the benefits that can be derived from a single layer of coating, a superior functional coating may be attained using more than one layer if the synergistic effects of the advantages of each layer can be realized. Bi- and multilayer coatings may be used for various purposes including enhancement of adhesion between the substrate and the top layer [4, 5], increase in hardness [6], and reduction of friction and wear [7]. The mechanism by which the coating system improves the surface property depends on the type of coatings as well as the deposition conditions including thickness, number of layers, and coating order. A coating architecture consisting of two or more layers may improve the load-carrying capacity by distributing the contact load over a larger area [8]. A soft coating on top of a hard coating may decrease the frictional force by providing a layer with low shear strength [9]. On the other hand, a hard coating on top of a soft coating may decrease wear by minimizing the probability of wear particle generation and plowing phenomenon [10]. Furthermore, the presence of multiple interfaces parallel to the substrate may impede dislocation movement or crack propagation, thereby increasing the hardness and toughness of the coating [11].

Bi- and multilayer coatings have been proposed and employed for tribological applications with various degrees of success. A dual-layer composite coating of carbon nanotubes (CNTs) and silver that was recently proposed to protect the surface of a silicon substrate showed a higher wear resistance compared to the single-layer silver or CNT coating [12]. However, the friction coefficient of the CNT/ silver dual-layer coating was relatively higher than those of 
the individual coatings. Likewise, the tribological characteristics of multilayer periodic nano-coatings based on carbon and boron nitride were investigated [13, 14]. These multilayer coatings exhibited a relatively low coefficient of friction and good wear resistance, but the deposition process of such coatings was quite complicated and difficult to control. In addition, residual stress that can lead to deformation of the substrate and delamination of the coating may result from the relatively large thickness of multilayer coating systems. Thus, though these and many other works have shown that bi- and multilayer coatings can be beneficial to improve some aspects of the tribological properties of a given substrate, plenty of opportunity still remains for further development and optimization of coating systems.

In this work, a new type of bi-layer coating was developed to provide low friction and high wear resistance for silicon-based materials. Specifically, the coating system consisted of amorphous silicon and amorphous carbon layers deposited on a silicon substrate. The bi-layer coating specimens were prepared with varying ratios of carbon and silicon layer thickness. The tribological properties of these coatings were compared to those of single-layer carbon and silicon coatings. The method of specimen fabrication and experimental details are described in the following sections.

\section{Experimental Details}

\subsection{Synthesis and Characterization of $\mathrm{C} / \mathrm{Si}$ Bi-layer Coatings}

To fabricate the $\mathrm{C} / \mathrm{Si}$ bi-layer coating on a silicon substrate, sputtering was selected as the coating process. The advantages of sputtering include good uniformity, high deposition rate, and wide variety of materials that can be processed. In addition, compressive residual stress can be induced in sputtered films which may be beneficial to decrease friction and wear by reducing the likelihood of tensile stress generation in the film during usage $[15,16]$. The bi-layer coating composed of an amorphous silicon layer deposited on an amorphous carbon layer that was first deposited on a silicon substrate. Amorphous silicon was chosen as the material for the upper layer because it is generally known to have a relatively low coefficient of friction [17]. Also, amorphous carbon was selected as the material for the bottom layer because it can be readily coated on a silicon substrate by sputtering with good adhesion. The thickness values of the individual layers in the coatings ranged from 10 to $100 \mathrm{~nm}$. It is important to note that amorphous carbon coating thicknesses greater than 100-150 nm often induce high levels of residual stress that can cause a blistering effect [18]. Thus, to maintain a residual stress to an appropriate level, the maximum thickness of the carbon layer was selected to be significantly less than $100 \mathrm{~nm}$. The residual stress level in sputtered silicon layer is generally lower than that of carbon, and therefore, relatively thick coatings may be deposited without negatively affecting the internal stress.

The $\mathrm{C} / \mathrm{Si}$ bi-layer and single-layer coatings were prepared by a dual DC magnetron sputtering process in an argon environment. The bi-layer coatings were deposited onto polished $\mathrm{Si}$ (111) wafers with an area of $1 \times 1 \mathrm{~cm}^{2}$. Graphite $(99.99 \%)$ and silicon $(99.999 \%)$ targets with diameters of $100 \mathrm{~mm}$ were used. The configuration of the deposition system is shown in Fig. 1, and Table 1 lists the specimens used in this work. The base pressure before the deposition was about $10^{-3} \mathrm{~Pa}$ and the argon pressure during deposition was maintained at $0.27 \mathrm{~Pa}$. The surface of the wafer was cleaned by an ion beam $(\mathrm{U} \approx 1000 \mathrm{~V}$, $\mathrm{I}=7 \mathrm{~mA}$ ) before deposition of the carbon layer. The desired level of coating thickness was achieved by properly setting the speed of the substrate holder using a stepper motor controlling system. A uniform coating could be obtained by moving the substrate above the shadow masks that were fixed above the targets as shown in Fig. 1. The substrate temperature was maintained below $50{ }^{\circ} \mathrm{C}$ during the sputtering process. The deposition rate was about 0.076 and $0.111 \mathrm{~nm} / \mathrm{s}$ for $\mathrm{C}$ and $\mathrm{Si}$, respectively.

Microstructures of the coatings were evaluated by lowangle X-ray diffraction (LAXRD) measurements made in the $\theta / 2 \theta$ geometry using a general-purpose X-ray diffractometer. $\mathrm{A} \mathrm{Cu}-\mathrm{K} \alpha_{1}$ wavelength of $0.15406 \mathrm{~nm}$ and an incidence radiation divergence of $0.1 \mathrm{mrad}$ were provided by an asymmetrically cut $\mathrm{Si}$ (110) crystal monochromator. The structure of the coatings was determined by calculating the theoretical diffraction pattern and comparing it with the experimental results. The diffraction patterns of the multilayers were analyzed based on the Fresnel equations [19] using the recurrence relation procedure reported in the literature $[20,21]$. Parameters of the individual layers of

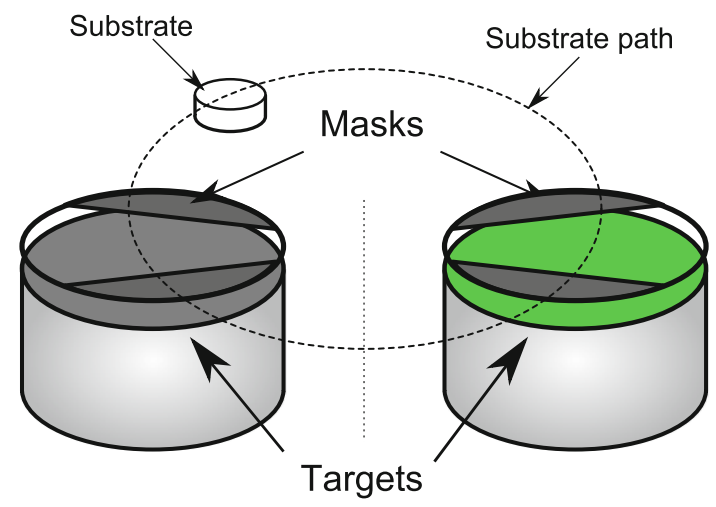

Fig. 1 Schematic of the carbon/silicon bi-layer coating deposition method using the DC magnetron sputtering system 
Table 1 List of specimens and composition of the coatings

\begin{tabular}{lll}
\hline Specimen name & Thickness, nm & \\
\cline { 2 - 3 } & Carbon & Silicon \\
\hline CSi-1 & 58 & 20 \\
CSi-2 & 58 & 51 \\
CSi-3 & 58 & 102 \\
CSi-4 & 10 & 102 \\
CSi-5 & 25 & 102 \\
CSi-6 & 100 & 100 \\
a-C & 50 & - \\
a-Si & - & 50 \\
\hline
\end{tabular}

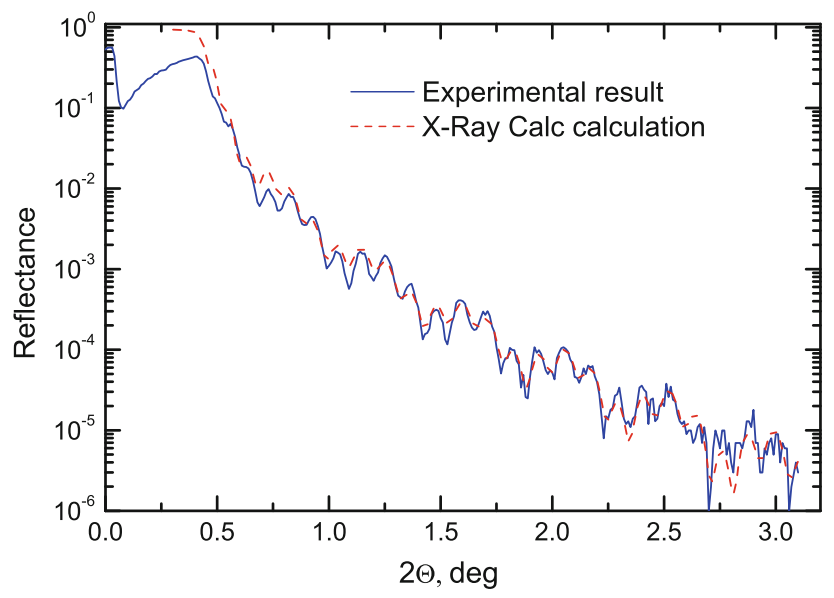

Fig. 2 Experimental low-angle X-ray diffraction and theoretically fitted curves of the bi-layer CSi-1 specimen

the multilayer coating model, such as thickness, density, and roughness, were varied until the best match between the calculated and the experimental diffraction curves was achieved [22]. An example of the experimental LAXRD and fitted theoretical curves for the bi-layer specimen CSi1 is shown in Fig. 2, and the fitting parameters for the theoretical model are shown in Table 2. During the sputtering of the top silicon layer, a thin interlayer (or mixing zone) formed between the carbon and silicon, causing a discrepancy between the LAXRD curve and the two-layer model. However, by introducing an intermixing layer composed of $\mathrm{SiC}$ at the $\mathrm{Si} / \mathrm{C}$ interface and using a density little less than that of the bulk, the experimental result could be modeled properly. Therefore, the actual structure of the $\mathrm{C} / \mathrm{Si}$ coating could be adequately described by a $\mathrm{C} / \mathrm{Si}_{\mathrm{x}} \mathrm{C}_{\mathrm{y}} / \mathrm{Si}$ three-layer model.

The chemical composition and surface morphology of the bi-layer coatings were investigated using a scanning electron microscope (SEM; Jeol 6210) equipped with an energy-dispersive X-ray spectroscopy (EDS; OXFORD INCA Energy) system and an atomic force microscope (AFM; Seiko SPA400), respectively. The AFM images
Table 2 Fitting parameters for LAXRD simulation of the bi-layer CSi-1 specimen

\begin{tabular}{lcll}
\hline Material & Thickness, $\mathrm{nm}$ & Density, $\mathrm{g} / \mathrm{cm}^{3}$ & Roughness, $\mathrm{nm}$ \\
\hline $\mathrm{Si}$ & 18.5 & 2.27 & 0.7 \\
$\mathrm{Si}_{\mathrm{x}} \mathrm{C}_{\mathrm{y}}$ & 0.8 & 2.50 & 0.6 \\
$\mathrm{C}$ & 54.3 & 2.00 & 0.5 \\
Substrate & - & 2.33 & 0.4 \\
\hline
\end{tabular}

were measured in contact mode at a sampling rate of 512 lines with 512 pixels per line using a silicon tip with a radius of $10 \mathrm{~nm}$. Figure 3 shows the electron micro-diffraction pattern obtained for the $\mathrm{C} / \mathrm{Si}$ bi-layer coating. Note that no diffraction rings are observed in the figure but only wide halos corresponding to amorphous materials are present. Thus, the structure of the $\mathrm{C} / \mathrm{Si}$ bi-layer coating was determined to be amorphous. This finding was also in agreement with other works which reported that sputtered silicon and carbon films have amorphous structures [22-24]. EDS analysis of the C/Si bi-layer coating surface showed that there was no particular contamination on the surface. The surface roughness of all the coating specimens measured using the AFM was found to be about $0.5-0.6 \mathrm{~nm}$.

\subsection{Tribological Investigation of the $\mathrm{C} / \mathrm{Si}$ Bi-layer Coatings}

The tribological behavior of the coatings was investigated using a commercial reciprocating tribo-tester (CETR UMT-2). Tests were performed in ambient conditions at a temperature of $\sim 24{ }^{\circ} \mathrm{C}$ and a relative humidity of $\sim 40 \%$. All of the tests were performed under applied normal loads in the range of $20 \sim 50 \mathrm{mN}$ and a sliding speed of $2 \mathrm{~mm} / \mathrm{s}$ with a $2 \mathrm{~mm}$ stroke. Stainless steel balls with a diameter of $1 \mathrm{~mm}$ were used as pins. At least three sliding tests were repeated for each experimental condition, and a new pin was used for each experiment. The total number of cycles ranged between 200 and 3,000, depending on the wear resistance of each specimen. All the experiments were performed in a class 100 clean room. The amount of wear was calculated from the $2 \mathrm{D}$ cross-sectional profile of the wear track obtained by the AFM.

The friction coefficient was recorded continuously during the sliding test for subsequent data processing. Figure 4 shows the average friction coefficient variation as a function of sliding cycles under an applied normal load of $20 \mathrm{mN}$ for the single-layer specimens, a-C (a) and a-Si (b), and for the bi-layer specimens, CSi-1 (c) and CSi-5 (d). The friction coefficient of the amorphous carbon at the initial stage of sliding was about 0.2 , but it continued to increase with increasing numbers of cycles and reached 


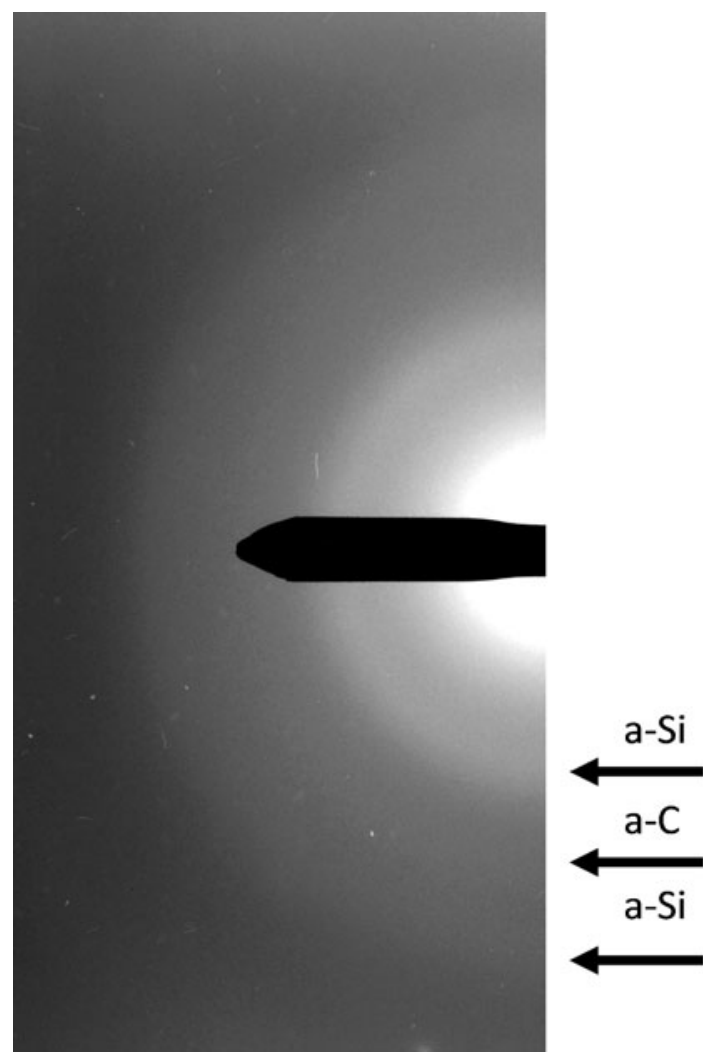

Fig. 3 Electron micro-diffraction image of the bi-layer $\mathrm{C} / \mathrm{Si}$ specimen

about 0.7 at the final stage of sliding. Such characteristics in the frictional behavior may be caused by the formation of wear particles which can result in severe plowing [25]. The sliding test was terminated after approximately 600 cycles because of the high wear rate. The friction coefficient of the single-layer silicon coating was significantly lower than that of the carbon (Fig. 4b), especially at the initial stage of sliding. In this case, the friction increased gradually during the first 200 cycles after which a sharp increase in the friction coefficient was observed. This abrupt increase in the friction coefficient was attributed to complete removal of the silicon coating due to wear.

The frictional behavior of the bi-layer coatings (Fig. 4c, d) was completely different from that of the single-layer coatings. In particular, the friction coefficient was relatively stable over a much longer sliding distance $(>900$ cycles). Moreover, the friction coefficient depended on the ratio of thickness between the carbon and silicon layers. Figure 5 presents a summary diagram of the friction coefficients of all the specimens for various applied normal loads. Under the low normal load of $20 \mathrm{mN}$, the friction coefficients of the single-layer carbon and silicon specimens were about 0.5 and 0.14 , respectively. When the normal load was increased to $50 \mathrm{mN}$, the friction coefficients of the single-layer carbon and silicon specimens increased to about 0.55 and 0.18 , respectively. Most of the bi-layer specimens had higher friction coefficients compared to the single-layer silicon specimen. Nevertheless, reducing the thickness of the carbon layer while maintaining the thickness of the silicon layer to about $100 \mathrm{~nm}$ led to a significantly lower friction coefficient. As shown in Fig. 5, the optimum coating for low friction was the bi-layer C/Si coating with a carbon thickness of $25 \mathrm{~nm}$. In the case of $20 \mathrm{mN}$ applied load, the friction coefficient was 0.09 , which was about $30 \%$ lower than that of the singlelayer silicon specimen.

The friction coefficient increased with increasing applied normal load but the degree of increase depended on the coating. Except for the single-layer carbon specimen, the rate of friction coefficient increase with respect to applied normal load was higher for specimens with relatively low friction coefficient under an applied load of $20 \mathrm{mN}$ (CSi-4, CSi-5, and a-Si). In this regard, CSi-5 that had the lowest friction coefficient under $20 \mathrm{mN}$, showed the greatest increase in the friction coefficient with respect to load.

The wear tracks of the specimens were characterized by SEM and AFM. Figure 6 shows the optical microscope and SEM images as well as the 2D AFM profiles of the wear tracks after the friction tests performed under an applied normal load of $20 \mathrm{mN}$. The skews that appear in the $2 \mathrm{D}$ profiles were due to surface leveling function of the AFM. As can be seen from the figure, the wear characteristics of the four specimens differed considerably. The single-layer carbon specimen showed the highest degree of surface damage (Fig. 6a). Despite the relatively short sliding distance, parts of the coating inside the wear track were completely removed. The wear track had several deep furrows penetrating down into the substrate, and the width of the track was about $30 \mu \mathrm{m}$. It was interesting to note that much of the wear debris was located slightly away from the boundary of the wear track, by about $10 \mu \mathrm{m}$. The region adjacent to the boundary of the wear track was relatively free from wear debris. The wear track formed on the single-layer silicon specimen (Fig. 6b) was also severe as the single-layer carbon specimen but the width of the wear track was smaller, being about $20 \mu \mathrm{m}$. Also, the wear debris was found along the boundary of the wear track. Other studies have shown that the presence of large delaminated areas inside the wear track and non-uniform spalling of the coating is typically observed with carbon coatings that have low adhesion to the substrate [26, 27]. Based on these reports and the wear pattern, it was postulated that the single-layer carbon coating had substantially lower adhesion to the substrate compared to the single-layer silicon coating.

The bi-layer CSi-1 specimen (Fig. 6c) showed wear characteristics similar to the single-layer silicon specimen, 

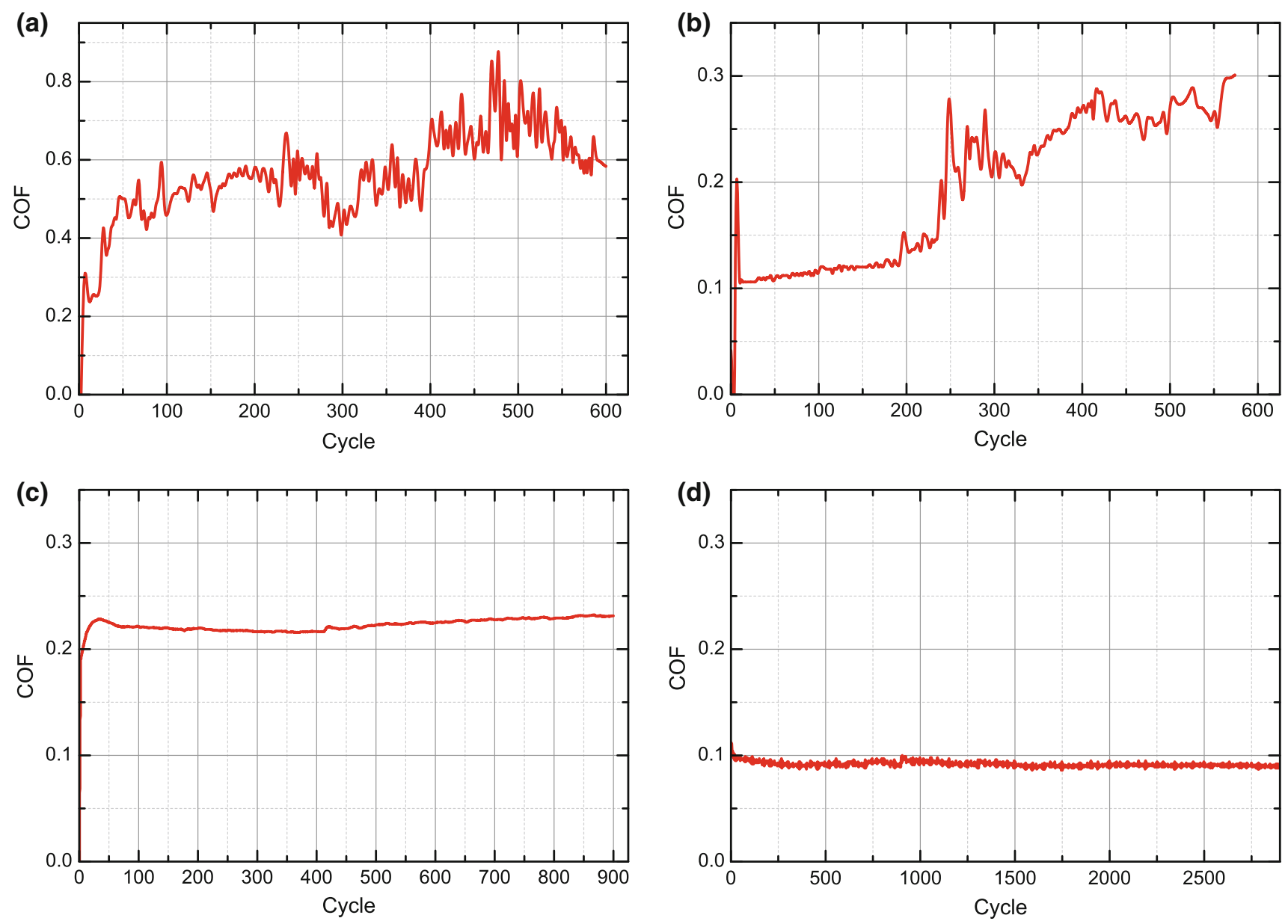

Fig. 4 Friction coefficient with respect to sliding cycles for (a) single-layer carbon, (b) single-layer silicon, (c) bi-layer CSi-1, and (d) bi-layer CSi-5 specimens. The friction tests were conducted using

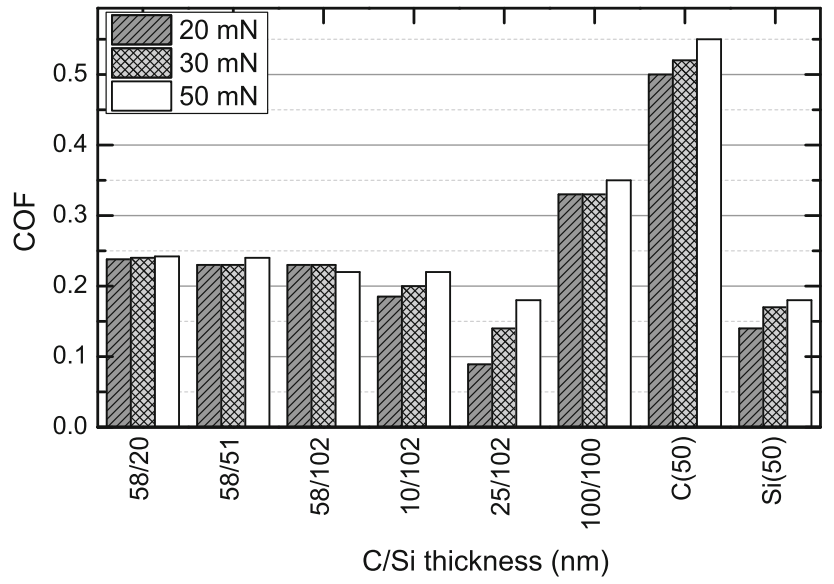

Fig. 5 Steady-state friction coefficients for the single- and bi-layer coating specimens obtained using a reciprocating-type tribo-tester under various applied normal loads with a sliding speed of $2 \mathrm{~mm} / \mathrm{s}$

except that the wear debris was located slightly away from the boundary of the track as in the case of the single-layer carbon specimen. The silicon coating was completely a reciprocating-type tribo-tester under an applied normal load of $20 \mathrm{mN}$ with a sliding speed of $2 \mathrm{~mm} / \mathrm{s}$

removed from the surface and the wear track width was about $30 \mu \mathrm{m}$. As for the wear of the bi-layer CSi-5 specimen, it can be seen from Fig. $6 \mathrm{~d}$ that the amount of wear was significantly less than that of the other three specimens even after a large number of sliding cycles $(>2,500)$. The wear track was not visible in the optical microscope image and the 2D AFM profile showed that the wear region was smoother than its surrounding which suggested that only burnishing wear occurred. When the applied normal load was increased to 30 and $50 \mathrm{mN}$ the wear characteristics of the CSi-5 specimen resembled those of the CSi-1 specimen.

The wear rates of all the specimens were calculated from the 2D AFM profiles of the wear tracks as shown in Fig. 7. The wear volume was estimated from the crosssectional area of the wear track below the horizontal line of the specimen surface. The product of the area and the sliding stroke was taken as the total wear volume. Since the wear tracks were irregular and shallow in depth, the area was calculated at multiple cross sections of the scanned 

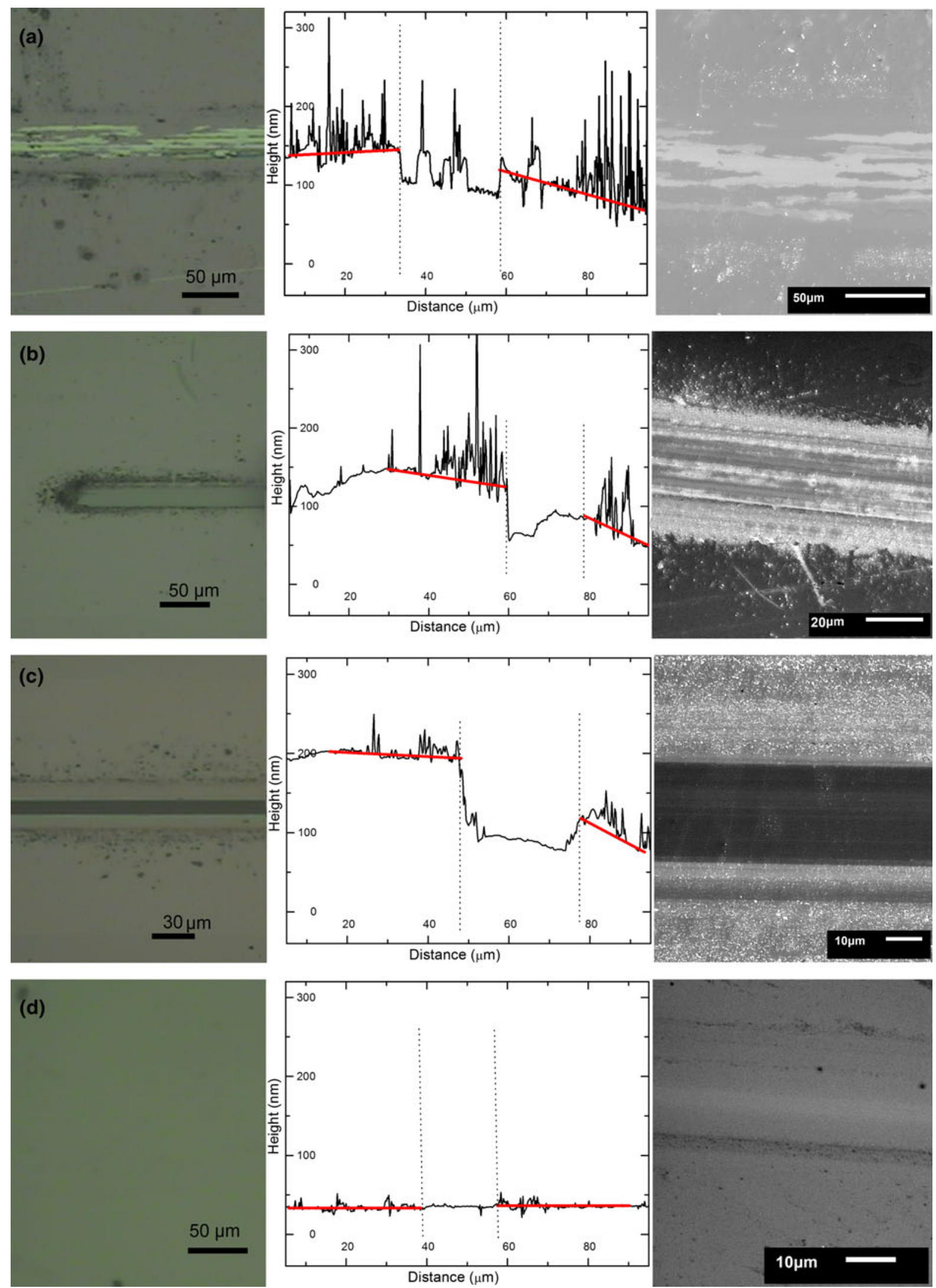

Fig. 6 Optical microscope and SEM images as well as 2D AFM profile of the wear track for (a) single-layer carbon, (b) single-layer silicon, (c) bi-layer CSi-1, and (d) bi-layer CSi-5 specimens 


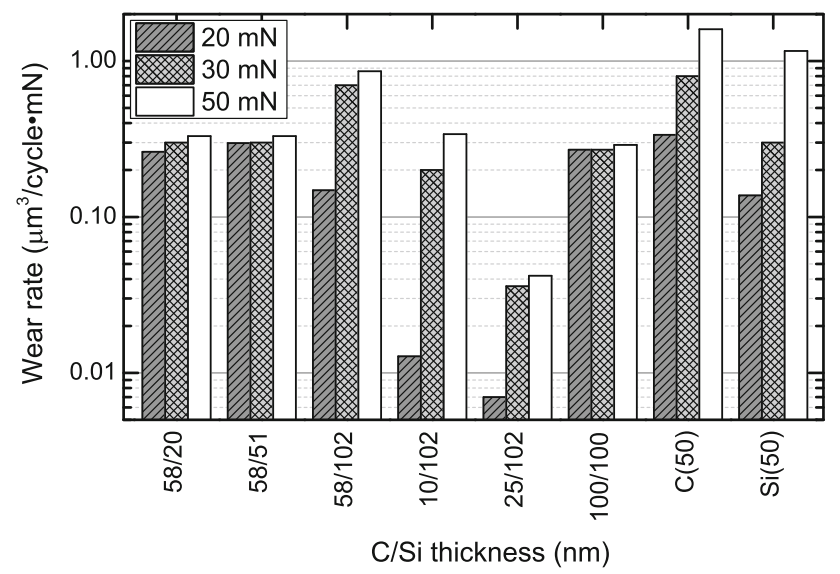

Fig. 7 Normalized wear rates of various coating specimens after the tribo-tests using a reciprocating-type tribo-tester under various applied normal loads with a sliding speed of $2 \mathrm{~mm} / \mathrm{s}$

region and averaged. The wear volume was then divided by the number of sliding cycles and the applied normal load to obtain the normalized wear rate.

It was found that the wear rate was strongly affected by the applied normal load. In case of the low load of $20 \mathrm{mN}$, the single-layer carbon specimen had the highest wear rate of $\sim 0.34 \mu \mathrm{m}^{3} /($ cycle $\cdot \mathrm{mN})$ and the bi-layer CSi-5 specimen with a carbon coating thickness of $25 \mathrm{~nm}$ had the lowest wear rate of $\sim 0.007 \mu \mathrm{m}^{3} /$ (cycle. $\mathrm{mN}$ ). The difference in the wear rate between the two specimens was about 48 times. Also, compared to the wear rate of the single-layer silicon specimen $\left(\sim 0.14 \mu \mathrm{m}^{3} /(\right.$ cycle $\left.\cdot \mathrm{mN})\right)$, the wear rate of the CSi-5 specimen was about 20 times lower. The wear rates of all the other bi-layer coating specimens with a carbon layer thickness greater than $25 \mathrm{~nm}$ were in between those of the single-layer carbon and single-layer silicon specimens. The bi-layer specimen with a carbon thickness of $10 \mathrm{~nm}$ had a wear rate of $\sim 0.013 \mu \mathrm{m}^{3} /($ cycle $\cdot \mathrm{mN}$ ) which was slightly higher than that of the CS-5 specimen but significantly lower than that of the single-layer silicon specimen.

As for the effect of the applied normal load on the wear rate, it was found that the wear rate either increased or did not vary significantly with increasing normal load depending on the coating. The wear rate increased with load for the single-layer specimens (a-C and a-Si) and bilayer specimens with $\mathrm{C} / \mathrm{Si}$ thickness ratios less than one (CSi-3, CSi-4, and CSi-5). Among all the specimens, CSi-5 had the lowest wear for all applied normal loads. Also, it was found that for $\mathrm{CSi}-4$ and $\mathrm{CSi}-5$ specimens with very low carbon thickness values, the percentage of wear rate increase as the load increased from 20 to $30 \mathrm{mN}$ was very significant. This finding suggested that there may be a transition in the wear mechanism of the specimen between these two loads. It was also noted that the wear rates of the single-layer specimens were significantly higher than all the bi-layer specimens at the highest applied normal load of $50 \mathrm{mN}$. Thus, the advantage of the bi-layer specimens regardless of their thickness ratios over the single-layer specimens in reducing the wear rate was clear for all normal loads used in this work.

\section{Mechanism of Friction and Wear Reduction of $\mathrm{C} / \mathrm{Si}$ Bi-layer Coatings}

The results of the tribological tests showed that the friction and wear characteristics of the sputtered $\mathrm{C} / \mathrm{Si}$ coating specimens were very much dependent on the thickness and composition of the coating layers. This was an expected outcome since the properties of the individual layers vary with deposition conditions. Based on the experimental results, a mechanism for the reduction of friction and wear of a $\mathrm{C} / \mathrm{Si}$ bi-layer coating is proposed. Figure 8 presents the wear mechanism models for the single-layer amorphous carbon (a), amorphous silicon (b), and bi-layer $\mathrm{C} / \mathrm{Si}$ (c) coatings. The schematics at the top show the contact situation between the upper pin and the coating specimen during the wear test, while the bottom ones depict the specimen conditions after the wear test with the pin removed.

Figure 8a depicts the model of wear for the single-layer carbon specimen. Under the applied normal load, the coating layer deforms by a relatively large amount due to its low stiffness. With repeated sliding the center region of the contact, where the pressure is the highest, experiences wear. The wear debris created along the wear track is naturally swept to the boundary of the track during subsequent sliding cycles as shown in the schematic. The wear debris gets accumulated slightly away from the wear track due to the relatively large elastic deformation of the contact region in the depth direction. Upon removal of the pin the elastically deformed zone returns to its original flatness. As can be seen in the SEM image of the wear track shown in Fig. 6a and illustrated in Fig. 8a, a distinct wear track is formed at the center and the wear debris remains at a slight distance away from the boundary of the wear track.

Figure $8 \mathrm{~b}$ depicts the model of wear for the single-layer silicon specimen. Compared to the case of the single-layer carbon specimen, the wear amount is lower and the wear debris is accumulated along the boundary of the wear track instead of being slightly away from it. This suggests that the amorphous silicon coating has a relatively higher stiffness than the amorphous carbon coating, and therefore, the degree of elastic deformation in the depth direction was much less under the applied normal load. Thus, upon removal of the load the wear debris remains along the boundary of the wear track as shown in the SEM image of Fig. $6 \mathrm{~b}$ and illustrated in Fig. $8 b$. 
(a)
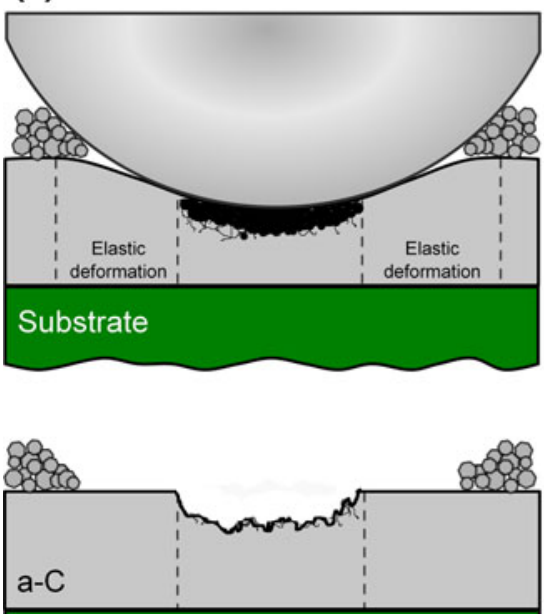

Substrate (b)
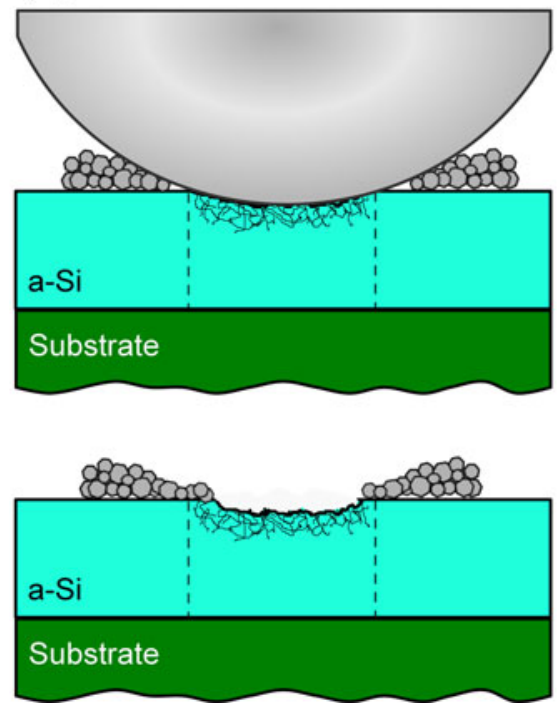

(c)
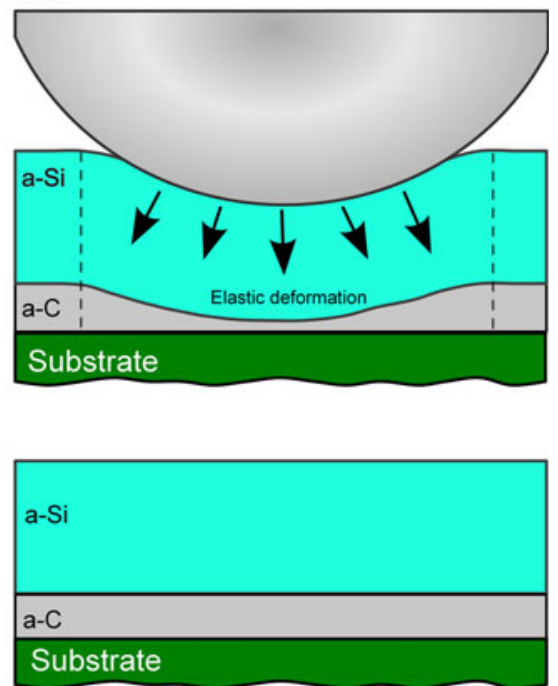

Fig. 8 Schematic (not to scale) of the wear mechanism models for the (a) single-layer carbon, (b) single-layer silicon, and (c) bi-layer $\mathrm{C} / \mathrm{Si}$ specimens. The schematics at the top show the contact situation

Finally, Fig. 8c depicts the model of wear for the bi-layer $\mathrm{C} / \mathrm{Si}$ specimen with the optimum thickness ratio. The relatively stiff and hard amorphous silicon coating is supported by a compliant amorphous carbon coating just below. Upon contact with the pin under the applied normal load the coating is elastically deformed sufficiently to distribute the load over a larger contact area. This allows the contact stress to be maintained below the critical stress level needed to cause significant wear. Also, the inherently low friction coefficient of the amorphous silicon coating can be achieved over a prolonged period since wear debris is not sufficiently generated to cause plowing. As can be seen in the SEM image of the wear track shown in Fig. 6d and illustrated in Fig. 8c, a distinct wear track is not formed and only evidence of slight burnishing wear can be found. Thus, with the optimum thickness ratio between the two coating layers, friction and wear can be minimized by lowering the interfacial shear stress and distributing the load over a larger area.

The wear model described above is primarily based on the experimental results obtained under an applied normal load of $20 \mathrm{mN}$ at which the best friction and wear properties are observed. However, the model can still be applied at higher normal loads while considering the fact that the wear rate increases significantly for the optimum bi-layer specimen (CSi-5). This may be explained by the reduction in the effective compliance of the underlying carbon layer due to greater degree of compression depicted in Fig. 8c. Thus, the overall wear reduction ability of the bi-layer coating diminishes at higher normal loads due to insufficient distribution of the contact load as predicted by the between the upper pin and the coating specimen during the wear test, while the bottom ones depict the specimen conditions after the wear test with the pin removed

model. It should be mentioned that the wear model proposed here is based on assumptions regarding the relative stiffness values of the a-C, a-Si, and C/Si bi-layer coatings. In reality it would be extremely difficult to measure the stiffness of these very thin coatings to confirm the validity of the proposed model. Nevertheless a reasonable explanation for the drastic decrease in the wear rate of the optimum bi-layer specimen confirmed by the experiments has been proposed and it may serve as a guideline to design other functional coatings for superior tribological properties.

\section{Conclusions}

The tribological behavior of carbon/silicon bi-layer coatings deposited on a silicon substrate by DC magnetron sputtering was assessed and compared to that of amorphous carbon and silicon coatings. Tribological tests were performed using a reciprocating tribo-tester under applied loads in the range of $20 \sim 50 \mathrm{mN}$ with a 1-mm-diameter steel ball used as the pin. The following conclusions may be drawn from the experimental results:

1. Under $20 \mathrm{mN}$ applied normal load, the friction coefficient of the bi-layer coating with 25-nm-thick carbon and 102-nm-thick silicon layers was 0.09 , which was significantly lower than that of the single-layer carbon and silicon coatings.

2. Under $20 \mathrm{mN}$ applied normal load, the wear rate of the bi-layer coating with 25-nm-thick carbon and 102-nm- 
thick silicon layers was about 48 and 20 times lower than that of the single-layer carbon and silicon coating, respectively.

3. The low friction and wear characteristics of the bilayer coating with the optimum thickness ratio was attributed to the inherently low frictional behavior of the top amorphous silicon coating and the distribution of contact load due to a relatively large elastic deformation which was made possible by the underlying compliant amorphous carbon coating.

4. The bi-layer amorphous carbon/silicon coating may be applied to attain the reliability and durability needed for implementation of various micro-scale devices with moving parts.

Acknowledgments This work was supported by a National Research Foundation of Korea (NRF) grant funded by the Korean government (MEST) (No. 2012-0001232).

\section{References}

1. Woo, Y., Kim, S.-H.: Sensitivity analysis of plating conditions on mechanical properties of thin film for MEMS applications. J. Mech. Sci. Tech. 25, 1017-1022 (2011)

2. Kim, H.-J., Kim, D.-E.: Nano-scale friction: a review. Int. J. Prec. Eng. Manuf. 10, 141-151 (2009)

3. Sung, I.-H., Kim, D.-E.: Surface damage characteristics of selfassembled monolayers of alkanethiols on metal surfaces. Trib. Lett. 17, 835-844 (2004)

4. Takeno, T., Sugawara, T., Miki, H., Takagi, T.: Deposition of DLC film with adhesive W-DLC layer on stainless steel and its tribological properties. Diamond Rel. Mater. 18, 1023-1027 (2009)

5. Voevodin, A., Schneider, J., Rebholz, C., Matthews, A.: Multilayer composite ceramic metal-DLC coatings for sliding wear applications. Trib. Int. 29, 559-570 (1996)

6. Miki, H., Takeno, T., Takagi, T.: Tribological properties of multilayer DLC/W-DLC films on Si. Thin Solid Films 516, 5414-5418 (2008)

7. Kim, D.-W., Lee, K.-Y., Jun, Y., Lee, S., Park, C.: Friction and wear characteristics of UHMWPE against $\mathrm{Co}-\mathrm{Cr}$ alloy under the wide range of contact pressures in lumbar total disc replacement. Int. J. Prec. Eng. Man. 12, 1111-1118 (2011)

8. Donnet, C., Martin, J.M., Le Mogne, T.H., Belin, M.: Super-low friction of $\mathrm{MoS}_{2}$ coatings in various environments. Trib. Int. 29, 123-128 (1996)

9. Holmberg, K., Ronkainen, H., Matthews, A.: Tribology of thin coatings. Ceramics Int. 26, 787-795 (2000)

10. Bull, S.J., Jones, A.M.: Multilayer coatings for improved performance. Surf. Coat. Techn. 78, 173-184 (1996)

11. Hogmark, S., Jacobson, S., Larsson, M.: Design and evaluation of tribological coatings. Wear 246, 20-33 (2000)
12. Kim, D.-E., Kim, C.-L., Kim, H.-J.: A novel approach to wear reduction of micro-components by synthesis of carbon nanotubesilver composite coating. CIRP Ann. Manuf. Tech. 60, 599-602 (2011)

13. Miyake, S., Hashizume, T., Kurosaka, W., Sakurai, M., Wang, M.: Deposition and tribology of carbon and boron nitride nanoperiod multilayer solid lubricating films. Surf. Coat. Techn. 202, 1023-1028 (2007)

14. Liu, D.G., Tu, J.P., Zhang, H., Chen, R., Gu, C.D.: Microstructure and mechanical properties of carbon nitride multilayer films deposited by DC magnetron sputtering. Surf. Coat. Techn. 205, 3080-3086 (2011)

15. Nix, W.D., Clemens, B.M.: Crystallite coalescence: a mechanism for intrinsic tensile stresses in thin films. J. Mater. Res. 14, 3467-3473 (1999)

16. Cuthrell, R.E., Mattox, D.M., Peeples, C.R., Dreike, P.L., Lamppa, K.P.: Residual stress anisotropy, stress control, and resistivity in post cathode magnetron sputter deposited molybdenum films. J. Vac. Sci. Technol. A 6, 2914-2921 (1988)

17. Zou, M., Cai, L., Wang, H., Yang, D., Wyrobek, T.: Adhesion and friction studies of a selectively micro/nano-textured surface produced by UV assisted crystallization of amorphous silicon. Trib. Lett. 20, 43-52 (2005)

18. Liu, L., Wang, Z., Zhu, J., Zhang, Z.: Intrinsic stress analysis of sputtered carbon films. Ch. Opt. Lett. 6, 384-385 (2008)

19. Jackson, J.D.: Classical electrodynamics, 2nd edn. John Wiley and Sons, New York (1975)

20. Attwood, D.T., Henke, B.L.: Low energy X-ray diagnostics. AIP Conf. Proc. 75, 280-285 (1981)

21. Barbee, T.W.: Multilayer X-ray optics. Opt. Eng. 25, 899-915 (1986)

22. Voronov, D.L., Zubarev, E.N., Kondratenko, V.V., Pershin, Yu.P., Sevryukova, V.A., Bugaev Ye, A.: Study of fast diffusion species in $\mathrm{Sc} / \mathrm{Si}$ multilayers by $\mathrm{W}$-based marker analysis. Thin Solid Films 513, 152-158 (2006)

23. Vinogradov, A.V., Uspenskii Yu, A., Artioukov, I.A., Pershin Yu, P., Zubaryev, E.N., Voronov, D.L., Penkov, O.V., Kondratenko, V.V., Seely, J.F.: Structure, thermal stability, and reflectivity of $\mathrm{Sc} / \mathrm{Si}$ and $\mathrm{Sc} / \mathrm{W} / \mathrm{Si} / \mathrm{W}$ multilayer X-ray mirrors. Proc. SPIE 4505, 230-236 (2001)

24. Artyukov, I.A., Bugayev, Ye.A., Devizenko Yu, O., Feschenko, R.M., Kasyanov Yu, S., Kondratenko, V.V., Romanova, S.A., Saveliev, S.V., Schfers, F., Feigle, T., Uspenski Yu, A., Vinogradov, A.V.: Carbon window soft X-ray imaging using multilayer optics. Proc. SPIE 5919, 59190 (2005)

25. Liu, Z., Sun, J., Shen, W.: Study of plowing and friction at the surfaces of plastic deformed metals. Trib. Int. 35, 511-522 (1995)

26. Knight, J., Page, T.: Interfacial phases and the adhesion of amorphous hydrogenated carbon films deposited by r.f. chemical vapour deposition onto (100) silicon. Surf. Coat. Tech. 53, 121-128 (1992)

27. Wang, Y., Li, H., Ji, L., Zhao, F., Kong, Q., Wang, Y., Liu, X., Quan, W., Zhou, H., Chen, J.: Microstructure, mechanical and tribological properties of graphite-like amorphous carbon films prepared by unbalanced magnetron sputtering. Surf. Coat. Tech. 205, 3058-3065 (2011) 